\title{
Constraints Based Decision Support for Site-Specific Preliminary Design of Wind Turbines
}

\author{
Abdelaziz Arbaoui ${ }^{1}$, Mohamed Asbik ${ }^{2}$ \\ ${ }^{1}$ M2I - ENSAM Meknès Ismaïlia, Meknès, Maroc \\ ${ }^{2}$ Laboratoire de Physique des Matériaux et Modélisation des Systèmes, Unité associée au CNRST-URAC, \\ Faculté des Sciences, Zitoune, Meknès, Maroc \\ E-mail: \{abdelaziz_arbaoui, asbik_m\}@yahoo.fr \\ Received April 7, 2010; revised May 21, 2010; accepted July 1, 2010
}

\begin{abstract}
This study presents a decision-support tool for preliminary design of a horizontal wind turbine system. The function of this tool is to assist the various actors in making decisions about choices inherent to their activities in the field of wind energy. Wind turbine cost and site characteristics are taken into account in the used models which are mainly based on the engineering knowledge. The present tool uses a constraint-modelling technique in combination with a CSP solver (numerical CSPs which are based on an arithmetic interval). In this way, it generates solutions and automatically performs the concept selection and costing of a given wind turbine. The data generated by the tool and required for decision making are: the quality index of solution (wind turbine), the amount of energy produced, the total cost of the wind turbine and the design variables which define the architecture of the wind turbine system. When applied to redesign a standard wind turbine in adequacy with a given site, the present tool proved both its ability to implement constraint modelling and its usefulness in conducting an appraisal.
\end{abstract}

Keywords: Wind Turbine, Decision Support, Preliminary Design, Cost Modelling, Constraint Satisfaction Problem (CSP), Digital CSP Solver

\section{Introduction}

For the past fifteen years, horizontal axis wind turbine systems (HAWT) have developed at a fast pace. Because of the renewability and cleanliness of the energy produced, incorporating such systems has become a key element in the new energy policies of many countries. Governments and non-trading companies show an important interest in sustainable development through the extensive incorporation of wind energy into electricity generation systems. Distributors are interested in the viability and in the cost as well as the quality of the energy produced. Aims of investors have been focalized on potential profits whereas designers, manufacturers and project managers define the architecture of the system and its fitness to the site.

Like all projects, a wind energy one is punctuated by successive phases with well-defined goals. In each phase, operations have to be performed and decisions have to be made by the various actors. Technical, economical, environmental and political issues lead the actors to justify their decision approach and search for decision-support means and tools. The main actors involved in the decision making process in the preliminary design phase are investors and distributors. To make a decision, these actors require external knowledge to their organisations. These are mainly within the competence of the project manager, manufacturer and scientist, and are needed to be translated into trends or estimations to be usable in the preliminary decision process. In addition, the characteristics of required data and models depend on the decision environment and inexpressible needs [1].

Various tools and software has been developed for wind energy systems. The objective of such tools is to maximise the performance and/or decrease the produced energy cost. Frequently, the strength properties and stresses of structures are all taken into account, with a finite-element and/or modal-analysis approach. Some tools use digital simulations to reproduce the aerodynamic characteristics of wind at the site. These ones focus on designing and defining details of wind energy systems, and they are not designed to provide decision support during the preliminary design phase [2]. 
This paper aims at presenting a knowledge base system for supporting decisions in the preliminary design of wind turbines. This tool is based on the development of a set of relations (called constraints) derived from engineering knowledge. Engineering knowledge has been related to the electrical energy production and the investment costs of the wind turbine systems.

The development of the knowledge base system has been performed through three main steps (see Figure 1):

- Analysis and structuring of the design problem,

- Development of a model relating to the design problem as a Constraint Satisfaction Problem,

- Implementation of the Constraint Satisfaction Problem on a digital CSP solver based on interval analysis [3].

The knowledge base system aims at exploring the solution space of the design problem. This exploration process is not an optimisation process since every solution satisfying the whole set of constraints of the problem is regarded as a solution. Provided that the size of the solution space is reasonably wide, the exploration process may be complete, namely, the solver delivers the complete set of solution of the problem. Therefore, decisionmakers are able to select wind turbines among a list. During this selection process, they are able to take into consideration some preferences resulting from their knowledge, which may be out of the scope of the model.

\section{Constraint Satisfaction Problem Solvers}

Digital processing tools of the Constraint Satisfaction Problem (CSP) solver type have recently been developed to cope with the difficulties presented by preliminary design. These tools are based on the notion of constraint, which converts the designer's knowledge into the form of conditions of compatibility between the variables of a design problem. Specific requirements of the industry, criteria of functional specifications and physical behaviour can all be described by the constraints. Generally, we call a Constraint Satisfaction Problem any problem that can be described in terms of a set of relationships

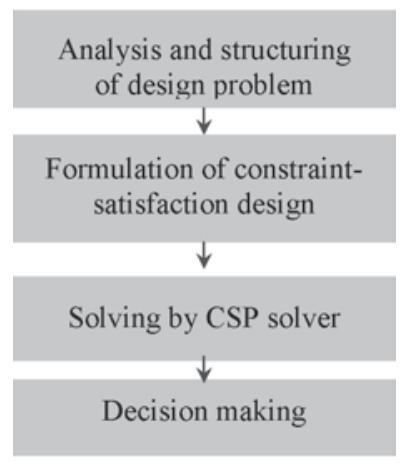

Figure 1. Applied approach. called constraints "C", variables "V" and domain values "D". Values assigned to the variables must belong to their respective domains while still satisfying problem constraints [4].

CSP solvers deal with problems integrating a large number of variables with values that evolve in the continuous space of real values. These variables represent dimensions, state variables (pressure, temperature, etc.) or performance criteria (costs, yields, etc.). The solvers are called digital CSP solvers. The sort of problems encountered in preliminary design also integrates variables that evolve in discrete domains, such as lists of concept, components or materials. Using mixed CSP solvers, continuous and discrete variables can be treated together.

The value domains assigned to the variables are intervals or unions of real intervals for the real value variables and enumerated sets or unions of integer intervals for the discrete variables. These domains can be left fairly broad so that no potential solutions to the design problem are eliminated.

The constraints traditionally used to represent the designer's input can be divided into three categories:

- Equal constraints (type "X $=\mathrm{Y}$ ”) usually represent laws of physics or definitions of performance criteria,

- Unequal constraints (type " $\mathrm{X}<\mathrm{Y}$ ”) usually represent economic constraints (costs), required space, etc.

- Logical constraints (type "W $\rightarrow$ (X and Y) or Z") represent conditional constraints such as technical skill rules, selection of components from catalogues, etc.

Constraints as used in constraint programming are relations which restrict the variable domains. The relationships that we take into account are algebraic ones which can integrate basic functions (trigonometric, logarithmic, etc.). A knowledge database is a file which indexes all the constraints and the domains assigned to the variables in a design problem. This database is digitally processed by a CSP solver which calculates the domain solutions for each variable that satisfies all the constraints in the problem.

In this study, we use the "Constraint Explorer®" software. This software was developed in the context of project CO2 (RNTL French Project "Conception par Contraintes”). It processes the knowledge bases in a twophase iterative and sequential alternant which gradually reduces and partitions the domains assigned to the CSP variables. The domains are gradually reduced until they satisfy the stipulations defined by the solver user. Figure 2 shows these phases in an example with two constraints and two variables. The variables take their values from intervals limited by real values.

The phases, in digital processing, alternate phases of propagating, the constraints and bisecting variable domains. Calculations converge towards an external approximation of the solution space, in other words, towards a set of value intervals assigned to the variables of the problem being modelled containing the solutions to the design problem. 


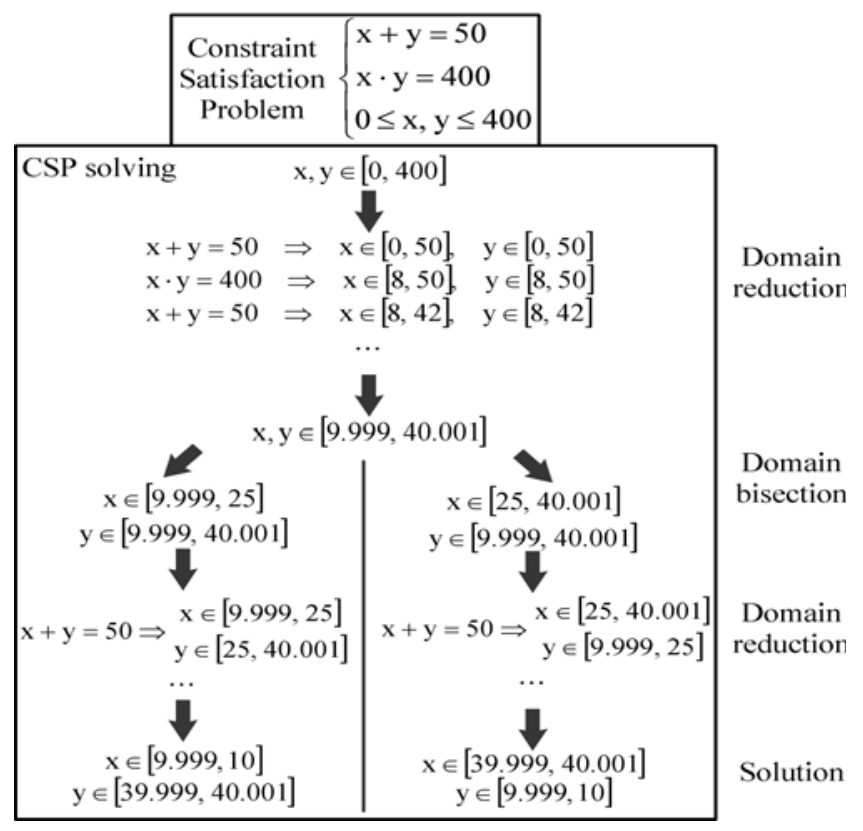

Figure 2. Constraint satisfaction problem solving taking into account 2 constraints and 2 variables.

This approach to solving Constraint Satisfaction Problems enables us to gradually limit the space containing design solutions, rather than testing different alternative solutions individually and validating them by simulating their functioning. This approach is extremely suitable for preliminary design problems where the aim is to select architectures for studied systems. Also, the solutions domain is explored in its entirety.

Digital processing moves towards a set of domains assigned to all the variables of the problem defining all the alternatives solutions to the design problem. Where "S" is the set of solutions to the design problem:

$$
S=\left\{S_{1}, \cdots, S_{i}, \cdots, S_{n}\right\}
$$

Each solution is a set of " $n$ " values assigned to " $m$ " variables " $V_{j}$ " of the problem:

$$
\forall i \in[1, n], \quad S_{i}=\left\{V_{1}^{i}, \cdots, V_{j}^{i}, \cdots, V_{m}^{i}\right\}
$$

Values assigned to variables are intervals for variables defined in real domains and integers for variables defined in integer domains:

$$
\begin{aligned}
& \forall i \in[1, n], \quad \forall j \in[1, m], \\
& \begin{cases}\text { If } V_{j}^{i} \text { is real : } & V_{j}^{i}=\left[\overline{V_{j}^{i}}{ }^{\min }, \overline{V_{j}^{i}} \max \right. \\
\text { If } V_{j}^{i} \text { is int eger }: & V_{j}^{i}=\overline{V_{j}^{i}}\end{cases}
\end{aligned}
$$

\section{Analysis and Structuring of HAWT Design Problem}

The wind turbine design problem gives rise to particular difficulties as wind turbines employ different technologies and concepts. Considering the multiplicity of potential choices, the interaction between the various parameters of the problem and the viewpoints to be taken into account, defining a wind turbine appropriate to the site proves quite difficult. In practice, these difficulties rise in anticipating and quantifying the consequences of a given choice. Such difficulties may result in an improper selection of the standard machine and lead to an omission of the potential profits guaranteed by a site specific design $[5,6]$.

Within the general category of horizontal axis wind turbines for grid applications there exists a great variety of possible rotor configurations, power control strategies and braking systems. Inevitably, there are situations in which decisions in one area can affect others. Alongside with these discrete design choices, there are several fundamental design variables, such as rotor diameter, machine rating and rotational speed, which also have to be established at the start of the design process. Continuous variables such as these lend themselves to mathematical optimization [7]. In this study, the following design variables are chosen to define a horizontal axis wind turbine:

- $\quad$ the nominal power, $P_{n}$

- $\quad$ the hub height, $H_{\text {hub }}$

- $\quad$ the rotor diameter, $D$

- $\quad$ the rotational speed, $N$

- $\quad$ the design speed, $V_{\text {des }}$

- $\quad$ the number of blades, $p$

- Control type: the present tool can be applied to constant-speed "stall" (CSS), constant-speed "pit- 
ch” (CSP), and variable-speed “pitch” (VSP) systems.

All of these design variables are often given in manufacturers catalogues.

The need related to the preliminary design of HAWT, consists in being able to characterize these configurations of design the ones compared to the others. We use the quality index, which is the ratio of the electricity produced on the total cost of the wind turbine, to choose the best solutions.

$$
Q I=\frac{E}{C_{W T}}
$$

To take safety problems into account, the distance between the tip of the blade and the ground should be equal to or more than $15 \mathrm{~m}$ :

$$
\frac{D}{2}+15 \leq H_{h u b}
$$

To limit aerodynamic noise from the rotor, blade tip linear speed cannot exceed $80 \mathrm{~m} / \mathrm{sec}$ :

$$
V_{\text {tip }}=\frac{2 \pi \mathrm{ND}}{120} \leq 80
$$

At this stage of problem definition, generating the constraints related to the cost of wind turbine and those related to the amount of energy produced is sufficient to start the solving phase.

\section{HAWT Cost Model}

The cost model of wind turbine encompasses the aspects related to the design and manufacture of such systems. It is the sum of cost models of the components of the wind turbine. A calibration factor $\mathrm{F}_{\mathrm{WT}}$ allows using real wind turbine costs [6].

$$
C_{W T}=F_{W T} \times \sum_{i} C_{\text {component }_{-} i}, \quad F_{W T}=1.1
$$

Flowcharts were used to identify the models cost of all components, Figure 3 for example, shows the flowchart used for the rotor.

The choice of level 2 in the flowchart of the rotor is justified to distinguish, firstly, the "pitch" and "stall" concept. In fact, the later encloses in his blade the tip braking mechanism, whereas the first contains the pitch mechanism in his hub. On the other hand, a two-blade rotor must contain the teeter mechanism to compensate his dynamic behavior.

The cost of some components is calculated from weight models developed using engineering estimation rules. These have been applied to the rotor, the transmission system, the nacelle, and the tower. As for the cost of the generator and associated electrical equipment, it is correlated with power rating. All models are calibrated (specific costs) to match the costs market of the components $[8,9]$.

\section{Annual Electricity Produced Model}

The amount of calculated electricity depends on the energy available on the site, at the level of the tower, the speed and geometric characteristics of the rotor, the output of the power unit, and the start/stop wind speeds of the wind turbine.

The wind in the site is defined as the following Weibull distribution:

$$
f(V)=\left(\frac{k}{V}\right) \cdot\left(\frac{V}{c}\right)^{k} e^{\left(-\frac{V}{c}\right)^{k}}
$$

Scale parameter $c$ characterises wind average speed, whereas shape parameter $k$ characterises wind distribution which varies with height [10]:

$$
k(Z)=k_{0}+0.03 Z+0.02
$$

where $k_{0}$ is the shape parameter at wind-measurement height $Z_{0}$.

The vertical gradient of wind speed is considered by introducing the following power law:

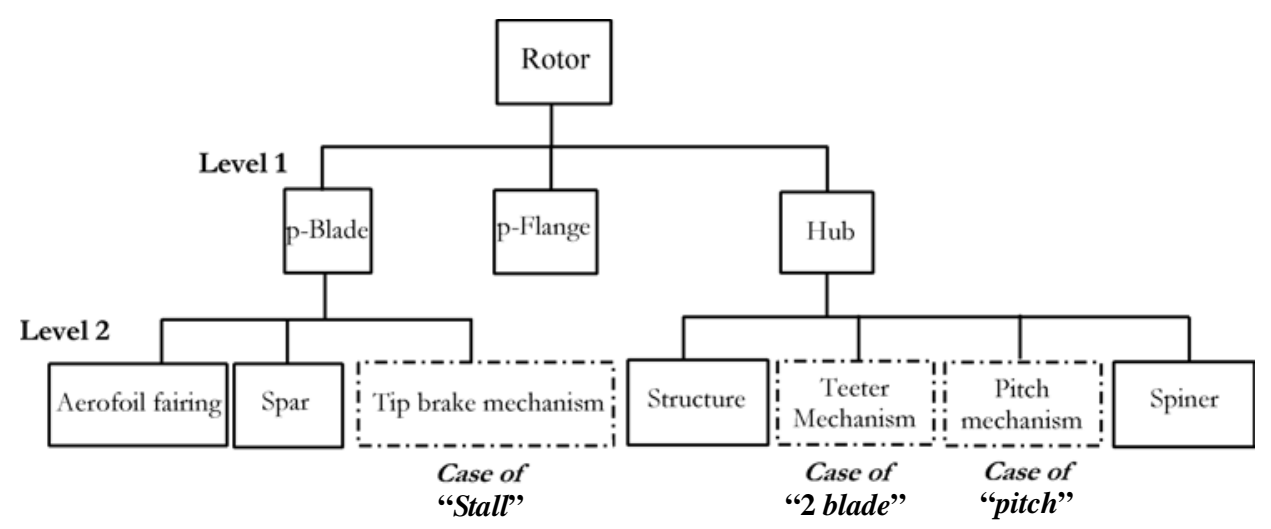

Figure 3. Flowchart of the rotor. 


$$
\frac{c}{c_{0}}=\left(\frac{Z}{Z_{0}}\right)^{\alpha}
$$

$c$ and $c_{0}$ are the scale parameters at heights $Z$ and $Z_{0}$ and $\alpha$ is considered constant.

The power recovered by a wind turbine is:

$$
P=\frac{1}{2} \rho \cdot C_{e} \cdot A \cdot V^{3}
$$

The efficiency factor depends on both wind speed and system architecture [2]:

$$
C_{e}(V)=C_{e m} \cdot \exp \left[-\frac{\left(\ln V-\ln V_{d e s}\right)^{2}}{2(\ln s)^{2}}\right]
$$

In this expression, the system is characterised by its maximum efficiency $C_{e m}$, its optimum operating speed $V_{\text {des }}$ (design speed), and its operating range $s$. The nominal power of the wind turbine is given by:

$$
P_{n}=\frac{1}{2} \rho C_{e m} A V_{d e s} \exp \left(\frac{9}{2}(\ln s)^{2}\right)
$$

$C_{e m}$ is calculated from the performance of the power conversion unit:

$$
C_{e m}=C_{p \max } \cdot \eta_{m} \cdot \eta_{g}
$$

The maximum value of $C_{p}$ is calculated using an analytical relationship [11]:

$$
\begin{aligned}
C_{P \max }= & 0.593 \cdot \\
& {\left[\begin{array}{l}
\frac{\lambda_{\max } p^{0.67}}{1.48+\left(p^{0.67}-0.04\right) \lambda_{\max }+0.0025 \lambda_{\max }^{2}} \\
-\frac{1.92 \lambda_{\max }^{2} p}{1+2 \lambda_{\max } p} \cdot \frac{C_{x}}{C_{z}}
\end{array}\right] }
\end{aligned}
$$

where

$$
\lambda_{\max }=\frac{\pi N D}{60 V_{d e s}}
$$

The efficiency of the gearbox is given by [9]:

$$
\eta_{m}=1-\left[\left(1-\pi_{m}\right)\left(\frac{P_{n}}{P}+3\right) / 4\right]
$$

with

$$
\pi_{m}=0.89 P_{n}^{0.012}
$$

The efficiency of the generator is given by [9]:

$$
\eta_{g}=1-\left[\left(1-\pi_{g}\right)\left(5\left(\frac{P \eta_{m}}{P_{n g}}\right)^{2}+1\right)\left(\frac{P_{n g}}{6 \eta_{m} P}\right)\right]
$$

with

$$
\pi_{g}=0.87 P_{n}^{0.014} \text {, }
$$

and

$$
P_{n g}=P_{n} \pi_{m} \pi_{g} F_{s}
$$

In this last expression, $F_{s}$ represents the service factor of the gearbox, which is defined by the following logical constraint:

$$
\left\{\begin{array}{c}
\text { Control type }=\text { CSS } \rightarrow F_{s}=2 \\
\text { Control type }=C S P \rightarrow F_{s}=1,75 \\
\text { Control type }=V S P \rightarrow F_{s}=1,25
\end{array}\right.
$$

Therefore, the annual electricity output in $\mathrm{kWh} /$ year of the wind turbine having a rotor with a surface area $A$, and the start/stop wind speeds $\left(V_{i}\right.$ and $\left.V_{f}\right)$, is the sum of the energies produced in one year (8,760 hours) which is reduced by the efficiency factor of the system $C_{e}(V)$ :

$$
E_{a p}=\frac{8.760 \cdot \rho}{2} \cdot A \cdot \sum_{V_{i}}^{V_{f}} f(V) \cdot C_{e}(V) \cdot V^{3} \cdot \Delta V
$$

\section{Preliminary Design of a Horizontal Wind Turbine}

The decision-making actors need the following data:

- The Criteria $(\mathrm{Cr})$ are the total cost of the wind system and the quantity of annual electricity produced. These two criteria allow the calculation of the quality index for a given configuration of wind turbine system.

- The design variables represent the parameters serving to define the architecture of the wind system $\left(P_{n}, H_{h u b}, D, N, V_{d e s}\right.$, Control type and $\left.p\right)$.

To define the relevance indicators of the solutions, the standard system VESTAS V39-500 is used. It corresponds to the ratio of the criteria values obtained by the total model on the values of criteria of the standard system.

$$
R I_{i}=\frac{C r_{i}}{C r_{s \tan d a r d}}
$$

The principle objective of the decision makers is to control the influence of design variables on the criteria. They often seek a machine which has a largest quality index but also which maximizes the annual produced electricity. Furthermore, the search for solutions (satisfaction of all the constraints) is carried out by using the “Constraint Explorer ${ }^{\circledR}$ " solver. According to the need, this tool can modify the fields of the design variables values and the variables which characterize the site.

In this study, a site whose characteristics are given in Table 1 has been chosen. To obtain a good judgement of total field of the solutions, we introduced the variation domain of the design variables gathered in Table 2. 
Table 1. Characteristics of the investigated site.

\begin{tabular}{cccc}
\hline$k_{0}$ & $c_{0}$ & $\alpha$ & $Z_{0}$ \\
\hline 1.2 & 8 & 0.12 & 30 \\
\hline
\end{tabular}

Table 2. Design variables and their domain of variation.

\begin{tabular}{cc}
\hline Design variable & Domain of variation \\
\hline$D(\mathrm{~m})$ & {$[20,80]$ with a step of $10 \mathrm{~m}$} \\
$P_{n}(\mathrm{~kW})$ & {$[400,2000]$ with a step of $100 \mathrm{~kW}$} \\
$V_{\text {des }}(\mathrm{m} / \mathrm{s})$ & {$[6,12]$ with a step of $2 \mathrm{~m} / \mathrm{s}$} \\
$H_{\text {hub }}(\mathrm{m})$ & {$[35,70]$ with a step of $10 \mathrm{~m}$} \\
$N(\mathrm{tr} / \mathrm{mn})$ & {$[15,50]$ with a step of $5 \mathrm{tr} / \mathrm{mn}$} \\
Control type & "PVC" or "SVC" or "PVV" \\
$P$ & 2 or 3 \\
\hline
\end{tabular}

Figure 4 shows the Pareto space of solutions obtained. It also shows some of the best solutions that we have chosen, in addition to standard system which appears as a solution of the problem, too. The best solutions chosen in Pareto front compared with the standard system are exhibited in Table 3.

These results reveal that an increase of the rotor diameter causes a diminution of the quality index. Furthermore, the increase of this geometrical parameter is associated with an important nominal power output, a weaker rotational speed and a higher tower. These results are in agreement with those of the reference [7].

We notice that all the given solutions in the table 3 are two blades with pitch variable speed control. We will return to justify this predominance in the continuation of this paper. Indeed, every solution of this table has a quality index clearly higher than that of the standard system for the studied site. Then, the relevance indicators associated with the four solutions are respectively: $142.8 \%$, $133.2 \%, 131.6 \%$, and $118 \%$. This means that the standard system is not adapted to the studied site and hence a redesign in adequacy with the site is necessary.

To improve the performances of the standard machine, we propose to deal with 6 possible redesign scenarios with which we highlighted the influence of the design variables on the performances of the wind system:

- Scenario 1 (Modification of the rotor): The design variables concerned with this scenario are the rotor diameter $D$ and the design speed $V_{\text {des }}$.

- Scenario 2 (Modification of the gearbox and the generator): This scenario relates to the nominal power output $P_{n}$ and the rotational speed $N$.

- Scenario 3 (Modification of the number of blade p)

- Scenario 4 (Modification of control type of the rotor): The objective is to compare a stall system with a pitch system.

- Scenario 5 (Modification of control type of the generator): The objective is to compare a constant speed system with a variable speed system.

- Scenario 6 (Modification of the whole wind system)

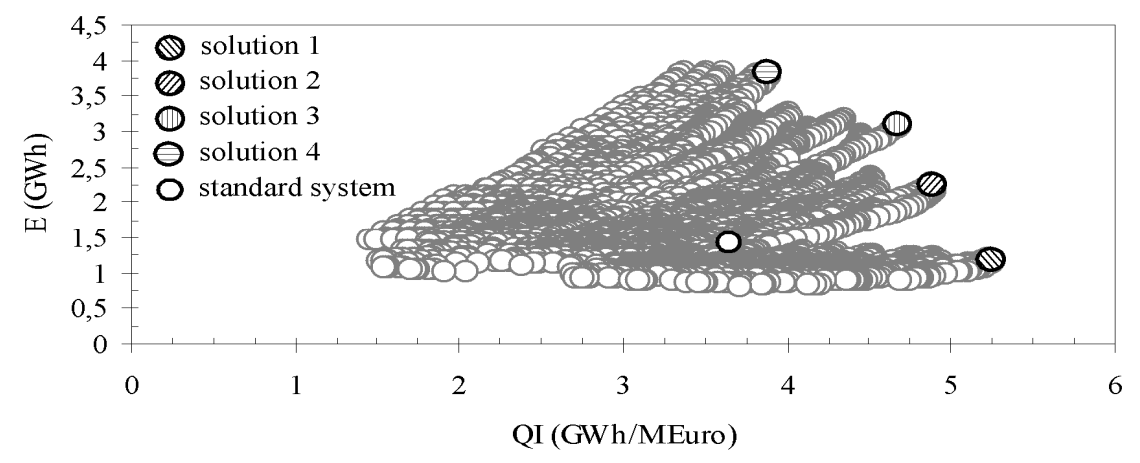

Figure 4. Field of solutions in the Pareto space.

Table 3. Criteria and design variables of standard system and some best solutions in Pareto front.

\begin{tabular}{ccccccccccc}
\hline \multirow{2}{*}{$\begin{array}{c}\text { Wind } \\
\text { Turbines }\end{array}$} & \multicolumn{3}{c}{ Criteria } & \multicolumn{1}{c}{ Design variable } \\
\cline { 2 - 11 } & $Q I$ & $E$ & $C_{W T}$ & $D$ & $P_{n}$ & $V_{\text {des }}$ & $H_{\text {hub }}$ & $N$ & Control type & $p$ \\
\hline Standard & 3.61 & 1.37 & 0.38 & 39 & 500 & 8 & 40.5 & 30 & CSP & 3 \\
Solution 1 & 5.24 & 1.19 & 0.23 & 30 & 700 & 10 & 35 & 46.8 & VSP & 2 \\
Solution 2 & 4.89 & 2.21 & 0.45 & 40 & 1200 & 10 & 45 & 33.9 & VSP & 2 \\
Solution 3 & 4.83 & 3.36 & 0.69 & 50 & 1700 & 10 & 45 & 24 & VSP & 2 \\
Solution 4 & 4.33 & 4.59 & 1.06 & 60 & 2000 & 10 & 55 & 20.9 & VSP & 2 \\
\hline
\end{tabular}


The optimal solutions (with respect to the quality index) obtained for each scenario are given in Table 4.

Thus, the optimal solution of the scenario 1 corresponds to the reduction of rotor diameter (18.5\%) and an increase in the design speed which reaches $12.5 \%$. As seen in Figure 5, there are also important opportunities to reduce the total cost of the wind system at the level of the nacelle, the tower and the foundation. These last opportunities must be seized by the decision-makers to be able to improve the quality index of their machine (to reach 4.12 GWh/MEuro instead of 3.67 GWh/MEuro which is equivalent to an indicator of relevance equal to $112.3 \%$ ). If we just reduce the rotor diameter the obtained quality index undergoes a lower reduction than that of the standard system (99\%). The gains which must be carried out at the level of the other components will certainly compensate the shortfall of the produced energy by a smaller rotor ( $82.5 \%$ only).

The retained solution of the scenario 2 provokes an increase of $60 \%$ in the nominal power and a weak reduction of $1.6 \%$ in the rotational speed. Then, the augmentation in the nominal power allows recovering more energy (126\%) and renders the wind system more expensive (an increase of 116\%). Figure 6 highlights that the rise of the wind system cost is not only due to the raised costs of the gearbox and the generator but also to the inevitable adaptation of the rotor and the nacelle. Indeed, the augmentation in the nominal power is accompanied by an increase in the weight supported by the nacelle and the tower and hence their costs. The rise of the hub and the flanges costs renders the rotor more expensive [9].

Table 4. Criteria and design variables of standard and best solutions for each scenario.

\begin{tabular}{|c|c|c|c|c|c|c|c|c|c|c|}
\hline \multirow{2}{*}{$\begin{array}{c}\text { Wind } \\
\text { Turbines }\end{array}$} & \multicolumn{3}{|c|}{ Criteria } & \multicolumn{7}{|c|}{ Design variable } \\
\hline & $Q I$ & $E$ & $C_{W T}$ & $D$ & $P_{n}$ & $V_{\text {des }}$ & $H_{h u b}$ & $N$ & Control type & $p$ \\
\hline Standard & 3.61 & 1.37 & 0.38 & 39 & 500 & 8 & 40.5 & 30 & CSP & 3 \\
\hline Scenario 1 & 4.12 & 1.13 & 0.27 & 31.8 & 500 & 9 & 40.5 & 30 & CSP & 3 \\
\hline Scenario 2 & 4.02 & 1.73 & 0.43 & 39 & 800 & 8 & 40.5 & 29.5 & CSP & 3 \\
\hline Scenario 3 & 3.88 & 1.36 & 0.35 & 39 & 500 & 8 & 40.5 & 30 & CSP & 2 \\
\hline Scenario 4 & 3.7 & 1.37 & 0.37 & 39 & 500 & 8 & 40.5 & 30 & CSS & 3 \\
\hline Scenario 5 & 3.91 & 1.37 & 0.35 & 39 & 500 & 8 & 40.5 & 30 & VSP & 3 \\
\hline Scenario 6 & 5.24 & 1.19 & 0.23 & 30 & 700 & 10 & 35 & 46.8 & VSP & 2 \\
\hline
\end{tabular}

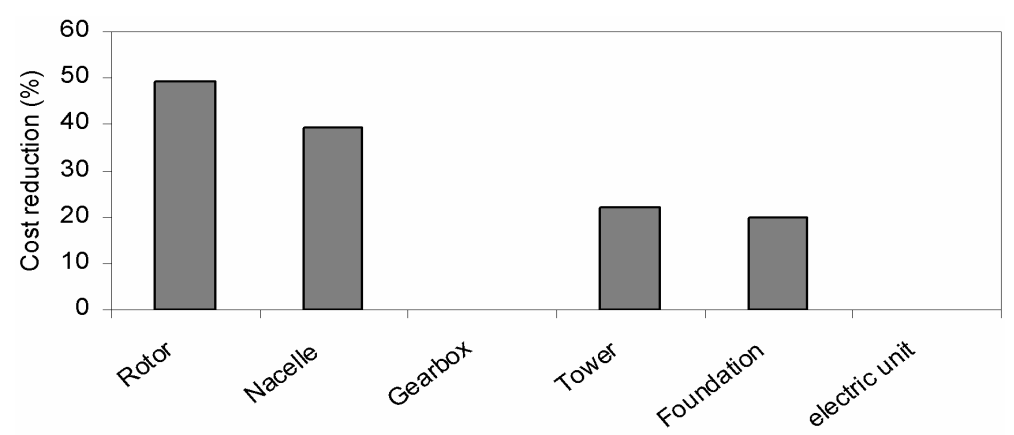

Figure 5. Cost reduction in scenario 1.

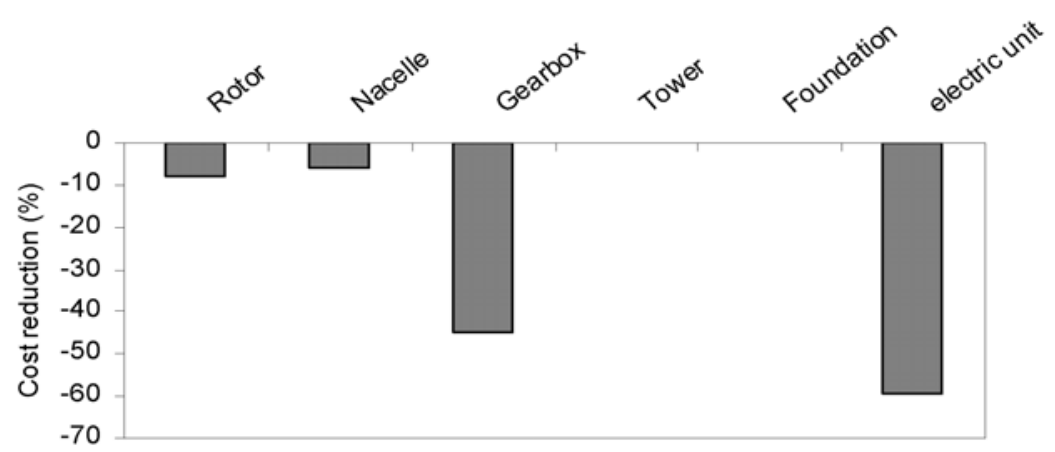

Figure 6. Cost reduction in scenario 2. 
Now, let us comment on scenarios 3, 4 and 5 concerning the discrete variables design. In fact, for the scenario 3 , a two-blade rotor is less heavy and hence less expensive although his blade is broader and thicker and that the teeter mechanism is integrated to his hub. The reduction of rotor weight leads to a weak reduction of the cost of the nacelle. The possibilities of reducing the cost of the two components are illustrated in Figure 7. According to the Equation (15), we can see that the power coefficient of a two-blade rotor is worse than that of threeblade system. This latter recovers more energy than the first rotor system but its production gain does not compensate its higher cost; so the quality index obtained is slightly lower than that of a two-blade system.

As for the scenario 4, a stall system seems to be the least expensive. This can be explained by the fact that its tip braking mechanism is less expensive than the pitch mechanism placed in the hub of the pitch system (see Figure 7). The use of a stall control raises the cost of the gearbox and generator. This is essentially due to the gearbox service factor which increases in the case of a stall system. On the other hand, the possible reduction in the rotor cost can not cover the rise in the gearbox and generator costs which gives a slightly higher quality index for stall system [9].

The gearbox and the electric unit play also an important role in the scenario 5 which relates to the control type of the generator. By using a variable speed control a decrease in the rotor cost becomes realistic, but the greatest part of this reduction is offered by the gearbox. This fact is due once again to the service factor of the gearbox which decreases in the case of variable speed system [9].

The last scenario has been examined as a combination of the previous ones. Then the wind system is considered as a two-blade system with a variable speed (scenarios 3 and 5). Its nominal power is higher than that of the standard system (scenario 2) whereas its rotor diameter is smaller (scenario 1). In Figure 8, the cost reduction of this case is exposed. In spite of the considerable increase in the electric unit cost, the possible gains on the level of the other components of the system allow to have a less expensive wind turbine. Furthermore, even if energy produced is reduced because of the reduction of the rotor diameter, the quality index is much improved.

Finally, the Table 5 recapitulates the gains in the quality index obtained for all the scenarios. These gains are more important for scenarios 1 and 2 (the design variables concerned are: $D, V_{\text {des }}, P_{n}$ and $N$ ) with comparison to scenarios 3, 4 and 5 which relate to the discrete design variables (control type and the number of blade). The profit reaches its maximum value for the scenario 6 , which represents a combination of the other scenarios.

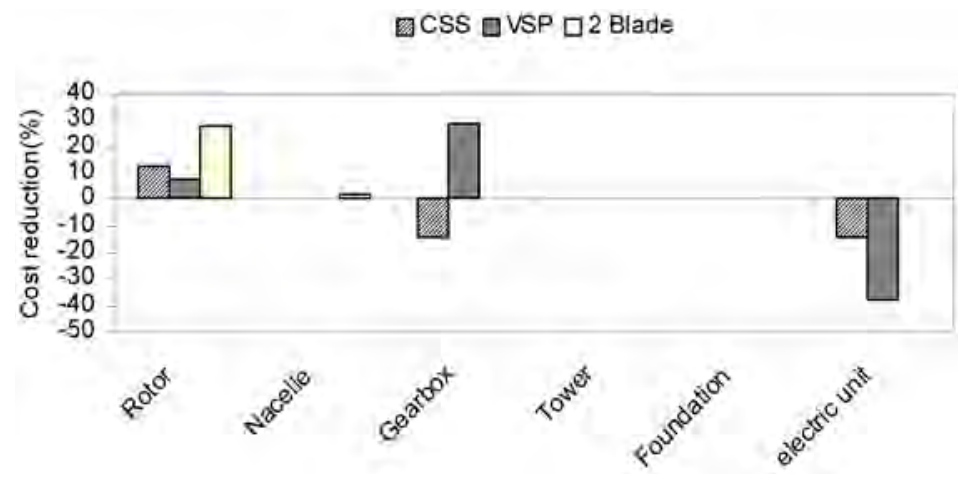

Figure 7. Cost reduction for scenarios 3, 4 and 5.

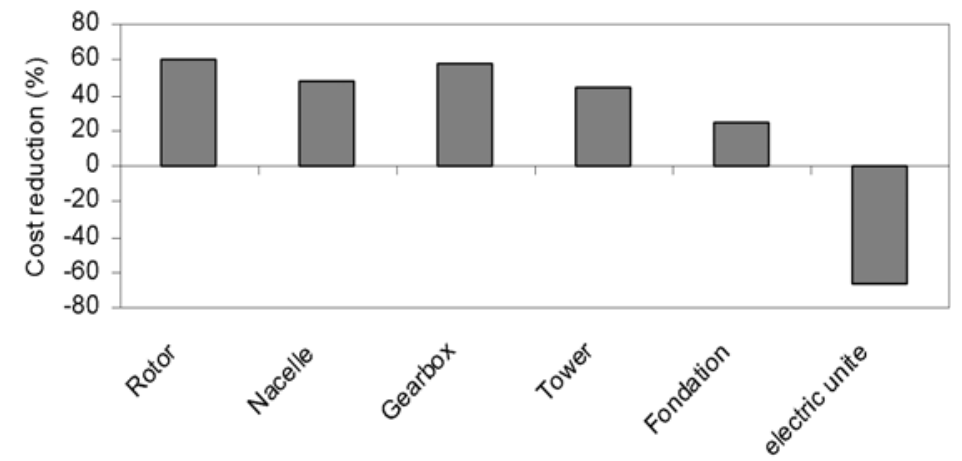

Figure 8. Cost reduction for scenario 6. 
Table 5. Profits in the quality index obtained for all the scenarios.

\begin{tabular}{lc}
\multicolumn{1}{c}{ Redesign Scenarios } & Gains \\
\hline Scenario 1: Modification of the rotor & $14 \%$ \\
Scenario 2: Modification of the gearbox and the generator & $11 \%$ \\
Scenario 3: Modification of the number of blade & $7.5 \%$ \\
Scenario 4: Modification of the control type of the rotor & $2.5 \%$ \\
Scénario 5: Modification of the control type of the generator & $8 \%$ \\
Scenario 6: Modification of the whole design variable & $45 \%$ \\
\hline
\end{tabular}

\section{Conclusions}

Decision support systems for the preliminary design of horizontal axis wind turbine is developed by taking into account the wind turbine components and site characteristics.

The present tool is mainly based on the engineering knowledge and it combines a constraint-modelling technique with a solving method derived from artificial intelligence (digital CSPs). In this way, it generates solutions and automatically performs the architecture selection and gives the cost of wind turbine components.

The present study highlights the relevance of the site specific design in the decision making process. The improvements achieved in terms of to the quality index are significant, this criteria is greatly affected by most of the design variables. When applied to redesign of standard wind turbine, our approach proved both its ability to implement constraint modelling and its usefulness to the various actors in conducting an appraisal.

\section{References}

[1] J. F. Courtney, "Decision Making and Knowledge Management in Inquiring Organization: Toward a New Decision-Making Paradigm for DSS," Decision Support Systems, Vol. 31, No. 1, 2001, pp. 17-38.

[2] C. T. Kiranoudis, N. G. Voros and Z. B. Maroulis, "Short-Cut Design of Wind Farms," Energy Policy, Vol. 29, No. 7, 2001, pp. 567-578.

[3] D. Scaravetti, J. Pailhès, J.-P. Nadeau and P. Sébastian,
“Aided Decision-Making for an Embodiment Design Problem: Advances in Integrated Design and Manufacturing in Mechanical Engineering,” Springer, Dordrecht, 2005.

[4] F. Benhamou and W. Older, "Applying Interval Arithmetic to Real, Integer and Boolean Constraints," The Journal of Logic Programming, Vol. 32, No. 1, 1997, pp. 124.

[5] P. Fuglsang, C. Bak, J. G. Schepers, T. T. Cockerill, P. Claiden, A. Olesen and R. Van Rossen, "Site-Specific Design Optimization of Wind Turbines," Wind Energy, Vol. 5, No. 4, 2002, pp. 261-279.

[6] T. Diveux, P. Sebastian, D. Bernard, J. R. Puiggali and J. Y. Grandidier, "Horizontal Axis Wind Turbine Systems: Optimization Using Genetic Algorithms,” Wind Energy, Vol. 4, No. 4, 2002, pp. 151-171.

[7] T. Burton, D. Sharpe, N. Jenkins and E. Bossanyi, "Wind Energy Handbook,” John Wiley \& Sons Ltd., London, 2001.

[8] A. Arbaoui, "Aide à La décision pour la définition d'un système éolien, Adéquation au site et à un réseau faible," $\mathrm{PhD}$ Thesis of the Ecole Nationale Supérieure d'Arts et Métiers de Bordeaux, 2006.

[9] R. Harrison and G. Jenkins, "Cost Modelling of Horizontal Axis Wind Turbines (Phase 2),” ETSU W/34/00170/ REP, University of Sunderland, 1994.

[10] I. Troen and E. L. Petersen, "European Wind Atlas," RISO, Commission of the European Communities, Risø National Laboratory, Roskilde, 1989.

[11] A. Spera, "Wind Turbine Technology," The American Society of Mechanical Engineering, New York, 1998. 


\section{Notations}

\begin{tabular}{|c|c|}
\hline$A$ & : rotor swept area $\left(\mathrm{m}^{2}\right)$ \\
\hline C & : Weibull distribution scale parameter $(\mathrm{m} / \mathrm{s})$ \\
\hline$C_{\text {component }}$ & : cost of component \\
\hline$C_{e}$ & : system efficiency factor \\
\hline$C_{e m}$ & : maximum system efficiency factor \\
\hline$C_{P}$ & : rotor power coefficient \\
\hline$C_{\text {pmax }}$ & : maximum power coefficient \\
\hline$C_{X}$ & : blade profile drag coefficient \\
\hline$C_{Z}$ & : blade profile lift coefficient \\
\hline$C_{W T}$ & : total cost of wind turbine (MEuros) \\
\hline$D$ & : rotor diameter $(\mathrm{m})$ \\
\hline$E$ & : annual electricity produced (GWh/year) \\
\hline$F_{S}$ & : service factor of gearbox \\
\hline$F_{W T}$ & : cost calibration factor \\
\hline$f$ & : Weibull distribution probability density \\
\hline$H_{\text {hub }}$ & : hub height (m) \\
\hline$k$ & : Weibull distribution shape parameter \\
\hline$N$ & : rotor rotation speed (rev/min) \\
\hline$p$ & : blade number \\
\hline$P_{n}$ & : nominal power $(\mathrm{kW})$ \\
\hline$P_{n g}$ & : generator power rating(kW) \\
\hline$Q I$ & : quality index \\
\hline$R I$ & : indicator of relevance \\
\hline$S$ & : operating range \\
\hline$V$ & : wind speed $(\mathrm{m} / \mathrm{s})$ \\
\hline$V_{\text {des }}$ & : design wind speed (m/s) \\
\hline$V_{f}$ & : network-disconnection speed (m/s) \\
\hline$V_{i}$ & : network-connection speed (m/s) \\
\hline$V_{\text {tip }}$ & : blade tip speed $(\mathrm{m} / \mathrm{s})$ \\
\hline \multicolumn{2}{|c|}{ Greek symbols } \\
\hline$\alpha$ & : wind shear factor \\
\hline$\lambda_{\max }$ & : maximum tip speed ratio \\
\hline$\rho$ & : air density $\left(\mathrm{kg} / \mathrm{m}^{3}\right)$ \\
\hline$\eta_{m}$ & : gearbox efficiency \\
\hline$\eta_{g}$ & : generator efficiency \\
\hline$\pi_{g}$ & : generator efficiency factor \\
\hline$\pi_{m}$ & : gearbox efficiency factor \\
\hline
\end{tabular}

